Hiroaki litsuka, Kun Li, Miyuki Kobayashi, Kikuko lida, Keiichi Noguchi, Noriyuki Yonezawa and Akiko Okamoto*

\title{
The crystal structure of $\left(1 R^{*}, 2 S^{*}\right)-1,2-b i s$ (2-fluorophenyl)-3,8-dimethoxyacenaphthene- 1,2-diol, $\mathrm{C}_{26} \mathrm{H}_{20} \mathrm{~F}_{2} \mathrm{O}_{4}$
}

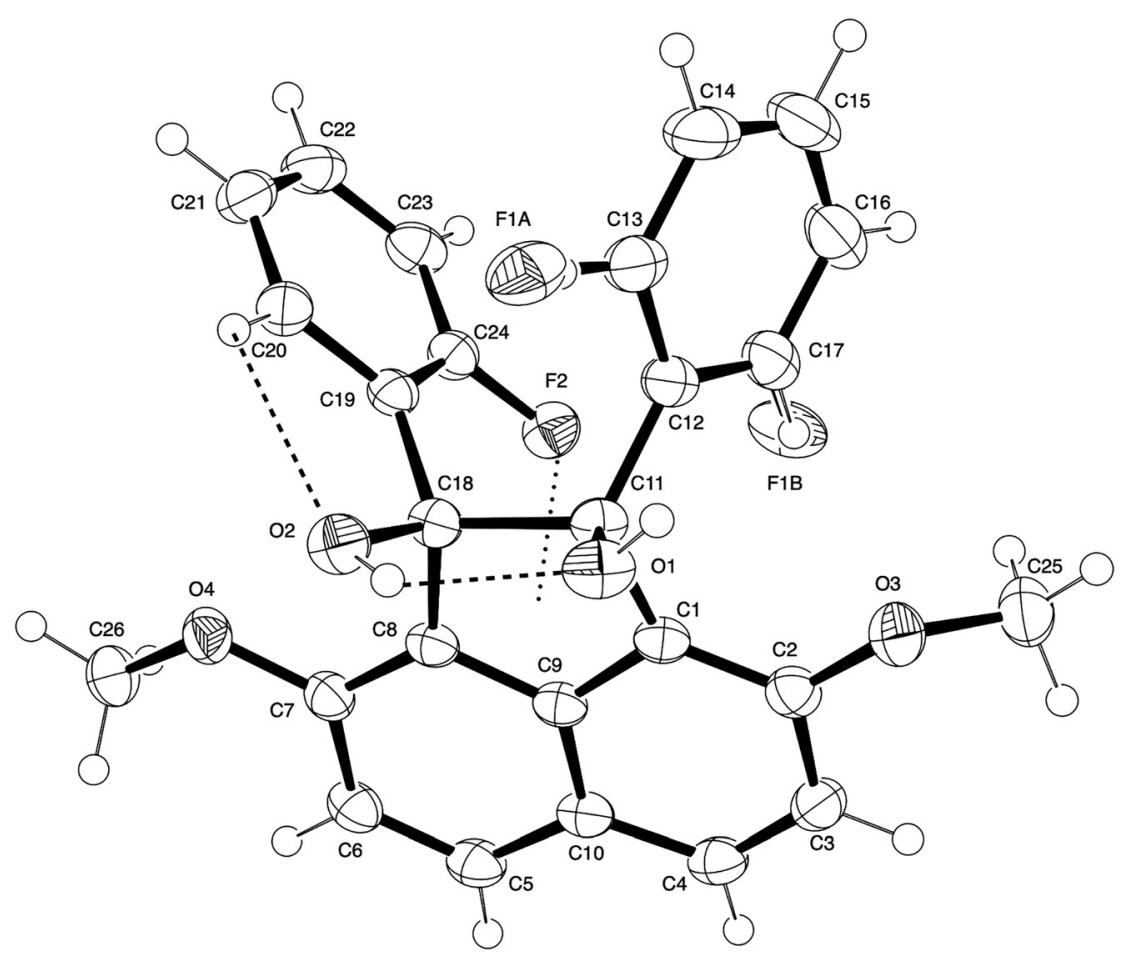

*Corresponding author: Akiko Okamoto, Department of Organic and Polymer Materials Chemistry, Tokyo University of Agriculture and Technology, 2-24-16 Naka-machi, Koganei, Tokyo 184-8588, Japan, E-mail: aokamoto@cc.tuat.ac.jp. https://orcid.org/0000-0002-41480798

Hiroaki litsuka, Kun Li, Miyuki Kobayashi and Kikuko lida, Department of Organic and Polymer Materials Chemistry, Tokyo University of Agriculture and Technology, 2-24-16 Naka-machi, Koganei, Tokyo 184-8588, Japan, E-mail: messhi.86583@gmail.com (H. litsuka), s212813v@st.go.tuat.ac.jp (K. Li),

m_matsukawa@yahoo.co.jp (M. Kobayashi),

kikumail@galaxy.dti.ne.jp (K. lida)

Keiichi Noguchi, Instrumentation Analysis Center, Tokyo University of Agriculture and Technology, 2-24-16 Naka-machi, Koganei, Tokyo 1848588, Japan, E-mail: knoguchi@cc.tuat.ac.jp

Noriyuki Yonezawa, Department of Organic and Polymer Materials Chemistry, Tokyo University of Agriculture and Technology, 2-24-16 Naka-machi, Koganei, Tokyo 184-8588, Japan; and Japan Association for Working Environment Measurement, 6th Floor, Mita Roudou Kijun Kyoukai Bldg, 4-4-5, Shiba, Minato-ku, Tokyo 108-0014, Japan, E-mail: yonezawa.noriyuki@jcom.zaq.ne.jp https://doi.org/10.1515/ncrs-2021-0314

Received August 1, 2021; accepted August 16, 2021; published online September 6, 2021

\section{Abstract}

$\mathrm{C}_{26} \mathrm{H}_{20} \mathrm{~F}_{2} \mathrm{O}_{4}$, monoclinic, $P 2_{1} / n$ (no. 14), $a=10.3707(2) \AA$, $b=12.0232(2) \AA, c=15.7744(3) \AA, \quad \beta=90.907(1)^{\circ}$, $V=1966.65(6) \AA^{3}, Z=4, R_{g t}(F)=0.0371, w R_{r e f}\left(F^{2}\right)=0.1013$, $\mathrm{T}=193.15 \mathrm{~K}$.

\section{CCDC no.: 2103419}

Table 1 contains crystallographic data and Table 2 contains the list of the atoms including atomic coordinates and displacement parameters.

\section{Source of material}

The title compound was prepared via $\mathrm{Zn}$-mediated reductive coupling reaction [6] of 1,8-bis(2-fluorobenzoyl)-2,7-dimeth- 
Table 1: Data collection and handling.

\begin{tabular}{ll}
\hline Crystal: & Colorless block \\
Size: & $0.35 \times 0.20 \times 0.10 \mathrm{~mm}$ \\
Wavelength: & Cu K $\alpha$ radiation $(1.54187 \AA)$ \\
$\mu:$ & $0.93 \mathrm{~mm}^{-1}$ \\
Diffractometer, scan mode: & Rigaku R-AXIS RAPID, $\omega$ \\
$\theta_{\text {max }}$, completeness: & $68.2^{\circ}, 99 \%$ \\
$N(h k l)_{\text {measured }}, N(h k l)_{\text {unique }}, R_{\text {int }}:$ & $36,085,3566,0.022$ \\
Criterion for $I_{\text {obs }}, N(h k l)_{\text {gt }}:$ & $I_{\text {obs }}>2 \sigma\left(I_{\text {obs }}\right), 3380$ \\
$N(\text { param })_{\text {refined }}:$ & 306 \\
Programs: & Rigaku [1, 2], SHELX [3, 4], ORTEP-III [5] \\
\hline
\end{tabular}

oxynaphthalene. The starting material was synthesized by reference to the literature [7]. In a $10 \mathrm{~mL}$ two-necked round-bottomed flask, 1,8-bis(2-fluorobenzoyl)-2,7-dimethoxynaphthalene ( $86 \mathrm{mg}, 0.2 \mathrm{mmol})$, zinc ( $78 \mathrm{mg}, 1.2 \mathrm{mmol})$, zinc chloride $(27 \mathrm{mg}, 0.2 \mathrm{mmol})$ and DMAc $(0.4 \mathrm{~mL})$ were stirred at $373 \mathrm{~K}$ under nitrogen atmosphere. After stirring for $4 \mathrm{~h}$, the reaction mixture was poured into water $(30 \mathrm{~mL})$. The resulting aqueous solution was extracted with chloroform (20 $\mathrm{mL} \times$ three times). The combined organic extracts were washed with $2 \mathrm{M} \mathrm{HCl} \mathrm{aq} \mathrm{(20} \mathrm{mL} \times$ three times) and brine (20 $\mathrm{mL} \times$ three times) successively. The organic layer thus obtained was dried over anhydrous $\mathrm{MgSO}_{4}$. The solvent was removed under reduced pressure to give a cake. Then the cake was dissolved in chloroform $(2 \mathrm{~mL})$ and the solution was added drop-wisely to hexane $(200 \mathrm{~mL})$ for reprecipitation. The precipitates were collected by suction filtration (quant.). Colorless block crystals of title compound were obtained by crystallization from ethyl acetate (68\% yield). ${ }^{1} \mathbf{H}$ NMR $\delta\left(300 \mathrm{MHz}, \mathrm{CDCl}_{3}\right): 8.09$ ( $1 \mathrm{H}$, dddd, $J=8.1,8.1,2.4$ and $2.4 \mathrm{~Hz}), 7.88(\mathrm{~d}, 1 \mathrm{H}, J=9.0 \mathrm{~Hz}), 7.77(\mathrm{~d}, 1 H, J=8.7 \mathrm{~Hz})$, $7.29(\mathrm{~d}, 1 H, J=9.0 \mathrm{~Hz}), 7.14(\mathrm{~d}, 1 H, J=8.7 \mathrm{~Hz}), 7.05(\mathrm{ddd}, 1 H$, $J=7.8,7.8$ and $1.5 \mathrm{~Hz}$ ), 7.02-6.88 (m, 3H), 6.76 (ddd, $1 H$, $J=7.8,7.8$ and $1.5 \mathrm{~Hz}$ ), 6.59 (ddd, $1 H, J=1,12.0$ and $0.9 \mathrm{~Hz}$ ), 6.25 (ddd, $1 H, J=11.4,7.8$ and $0.9 \mathrm{~Hz}$ ), 4.48 (s, $1 H$ ), 4.31 (d, $1 H, J=6.6 \mathrm{~Hz}), 3.90(\mathrm{~s}, 3 H), 3.66(\mathrm{~s}, 3 H) \mathrm{ppm} ;{ }^{13} \mathbf{C}$ NMR $\delta$ $\left.\left(75 \mathrm{MHz}, \mathrm{CDCl}_{3}\right): 160.79 \mathrm{~J}_{C-F}=247 \mathrm{~Hz}\right), 158.67$ $\left(J_{C-F}=246 \mathrm{~Hz}\right.$ ), 153.53, 153.09, 139.44 (pseudo s, $J_{C-F}=2.9$ $\mathrm{Hz}), 130.73\left(J_{C-F}=10.1 \mathrm{~Hz}\right), 130.66,129.43\left(U_{C-F}=8.6 \mathrm{~Hz}\right)$, $128.73\left(J_{C-F}=7.9 \mathrm{~Hz}\right), 128.34,128.02,127.94\left(J_{C-F}=12.2 \mathrm{~Hz}\right)$, $127.32\left(J_{C-F}=7.2 \mathrm{~Hz}\right), 127.16,123.76,123.00\left(J_{C-F}=2.8 \mathrm{~Hz}\right)$, $122.53\left(J_{C-F}=2.8 \mathrm{~Hz}\right), 122.17,115.17\left(J_{C-F}=23.0 \mathrm{~Hz}\right), 114.43$, $114.10\left(J_{C-F}=23.0 \mathrm{~Hz}\right.$ ), 113.61, 89.95 (pseudo s), 85.66 $\left(J_{C-F}=2.85 \mathrm{~Hz}\right), 56.56,56.16 \mathrm{ppm}$. IR (KBr): $3505(\mathrm{O}-\mathrm{H})$, $3464(\mathrm{O}-\mathrm{H}), 1630$ (Ar), 1581 (Ar), 1504 (Ar), 1485, 1225, $1040 \mathrm{~cm}^{-1}$. HRMS $(\mathrm{m} / z):\left[\mathrm{M}+\mathrm{H}-\mathrm{H}_{2} \mathrm{O}\right]^{+}$calcd. for $\mathrm{C}_{26} \mathrm{H}_{19} \mathrm{~F}_{2} \mathrm{O}_{3}$, 417.1302, found, 417.1318. m.p. $=450-451 \mathrm{~K}$.
Table 2: Fractional atomic coordinates and isotropic or equivalent isotropic displacement parameters $\left(\AA^{2}\right)$.

\begin{tabular}{|c|c|c|c|c|}
\hline Atom & $x$ & $y$ & $z$ & $U_{\text {iso }}{ }^{*} / U_{\text {eq }}$ \\
\hline $\mathrm{C} 1$ & 0.98369 (13) & $0.31411(11)$ & $0.17894(8)$ & $0.0258(3)$ \\
\hline $\mathrm{C} 2$ & 07530 (13) & 39373 (12) & $.19528(9)$ & $.0291(3)$ \\
\hline 3 & 20809 (14) & 36570 (12) & $0.18799(9)$ & $.0325(3)$ \\
\hline H3 & 1.2716 & 0.4212 & 0.1985 & $0.039^{\star}$ \\
\hline $\mathrm{C} 4$ & 24645 (13) & $0.26042(13)$ & $0.16621(9)$ & $0.0322(3)$ \\
\hline H4 & 1.3357 & 0.2446 & 0.1607 & $0.039^{\star}$ \\
\hline 5 & $1.17750(13)$ & $.06201(12)$ & $0.13210(9)$ & 0.0327 (3) \\
\hline H5 & 1.2636 & 0.0361 & 0.1276 & $0.039^{\star}$ \\
\hline $\mathrm{C} 6$ & $1.07676(14)$ & $0.01071(12)$ & $0.11942(9)$ & $0.0320(3)$ \\
\hline H6 & 1.0950 & -0.0861 & 0.1063 & $0.038^{*}$ \\
\hline C7 & $0.94611(13)$ & $0.02378(11)$ & $0.12541(9)$ & 0.0277 (3) \\
\hline C8 & $0.92073(12)$ & 11) & $37(8)$ & $0.0254(3)$ \\
\hline C9 & $1.02378(13)$ & 1) & $80(8)$ & 0.0257 (3) \\
\hline C10 & $2(13)$ & 2) & $6(9)$ & $0.0286(3)$ \\
\hline C11 & $0.83781(13)$ & $0.31780(11)$ & $0.18745(8)$ & $0.0262(3)$ \\
\hline C12 & $0.76872(13)$ & $0.40893(11)$ & $0.13776(9)$ & $0.0293(3)$ \\
\hline $13^{a}$ & $0.63866(14)$ & $0.43100(13)$ & $0.14949(10)$ & $0.0368(3)$ \\
\hline 13 & 0.5923 & 58 & 0.1879 & $.044^{\star}$ \\
\hline 14 & $0.57381(18)$ & 51553 & 10789 (13) & $.0512(5)$ \\
\hline 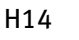 & 0.4852 & 0.5288 & 0.1183 & $.061^{*}$ \\
\hline C15 & $0.6396(2)$ & 15) & 091 (13) & $.0562(5)$ \\
\hline 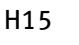 & 963 & & 0.0218 & $.067^{*}$ \\
\hline C16 & 0.76816 (19) & $.56041(14)$ & $0.03620(11)$ & $.0473(4)$ \\
\hline H16 & 0.8133 & 0.6047 & 0034 & $.057^{\star}$ \\
\hline $\mathrm{C} 17^{\mathrm{b}}$ & $0.83134(16)$ & $0.47555(12)$ & $0.07931(10)$ & 0.0355 (3) \\
\hline H17 & .9200 & & 686 & $.043^{*}$ \\
\hline C18 & $0.79264(12)$ & 1) & $9(8)$ & $0.0256(3)$ \\
\hline C19 & 0.70 & & (9) & $4(3)$ \\
\hline 20 & 13) & 2) & (10) & $0.0318(3)$ \\
\hline $\mathrm{H} 2 \mathrm{O}$ & & & 371 & $038^{\star}$ \\
\hline 21 & $0.50367(15)$ & 3599 (13) & $.01195(11)$ & $.0380(4)$ \\
\hline $\mathrm{H} 21$ & 0.4187 & & 158 & $.046^{*}$ \\
\hline $\mathrm{C} 22$ & $0.54886(15)$ & 17018 (13) & $0.06560(10)$ & $.0392(4)$ \\
\hline רa & & & & $.047^{\star}$ \\
\hline C23 & 67255 & 2) & 0.070 & $0.0343(3)$ \\
\hline H23 & 52 & 75 & 236 & $0.041^{\star}$ \\
\hline $\mathrm{C} 2$ & & & (9) & 0.0277 (3) \\
\hline $\mathrm{C} 25$ & 37 (18) & 3) & (12) & $0.0463(4)$ \\
\hline & 1.0631 & & 0.2079 & $0.056^{*}$ \\
\hline H25 & 911 & 2 & 1970 & $0.056^{*}$ \\
\hline $\mathrm{H} 25 \mathrm{C}$ & & & 30 & $0.056^{\star}$ \\
\hline $\mathrm{C} 2$ & $0.86957(15)$ & $0.15402(12)$ & $.07840(10)$ & $0.0342(3)$ \\
\hline $\mathrm{H} 26 \mathrm{~A}$ & 0.7886 & -0.1954 & 0.0721 & $0.041^{*}$ \\
\hline H26B & 0.9098 & -0.1464 & 229 & $0.041^{*}$ \\
\hline $\mathrm{H} 26 \mathrm{C}$ & 0.9280 & -0.1942 & 0.1170 & $0.041^{\star}$ \\
\hline $\mathrm{F} 1 \mathrm{~A}^{\mathrm{b}}$ & $0.57062(9)$ & $0.36665(9)$ & $0.20276(7)$ & 0.0457 (3) \\
\hline $\mathrm{F} 1 \mathrm{~B}^{\mathrm{a}}$ & $0.9303(15)$ & $0.4543(14)$ & $0.0459(9)$ & $0.043(4)$ \\
\hline F2 & $0.86876(8)$ & $0.26034(7)$ & $-0.00543(5)$ & $0.0346(2)$ \\
\hline 01 & $0.81215(10)$ & $02(10)$ & $0.27618(6)$ & $0.0338(2)$ \\
\hline $\mathrm{H} 1$ & $0.783(2)$ & $0.3883(19)$ & 0.2849 (14) & $0.060(7)^{\star}$ \\
\hline 02 & 72549 (10) & $0.14048(9)$ & $0.22742(7)$ & $0.0334(2)$ \\
\hline $\mathrm{H} 2$ & $0.746(2)$ & 0.1767 (19) & $0.2702(15)$ & $0.060(7)^{\star}$ \\
\hline
\end{tabular}


Table 2: (continued)

\begin{tabular}{lrrrr}
\hline Atom & $\boldsymbol{x}$ & $\boldsymbol{y}$ & $\boldsymbol{z}$ & $\boldsymbol{U}_{\text {iso }} \boldsymbol{U}_{\text {eq }}$ \\
\hline 03 & $1.03595(10)$ & $0.49682(8)$ & $0.22181(7)$ & $0.0367(3)$ \\
04 & $0.84373(9)$ & $-0.04593(8)$ & $0.11230(7)$ & $0.0321(2)$ \\
\hline
\end{tabular}

Occupancy: $0.06,{ }^{b}$ Occupancy: 0.94 .

\section{Experimental details}

All $\mathrm{H}$ atoms were found in a difference map and were subsequently refined as riding atoms, with $\mathrm{C}-\mathrm{H}=0.95$ (aromatic) and 0.98 (methyl) $\AA$, and with $U_{\text {iso }}(\mathrm{H})=1.2 U_{\text {eq }}(\mathrm{C})$.

\section{Comment}

Weak interactions such as non-classical hydrogen bond [8-11] are ordinarily latent, however, crystals of noncoplanarly accumulated aromatic rings compounds can expectingly pull out weak interactions by depressing $\pi \cdots \pi$ stacking. Such a skeleton is embodied in title pinacol, which is reductively coupling product of peri-aroylnaphthalene compounds [12-17]. In the title molecular structure, two 2-fluorophenyl groups are oriented in a same direction against the acenaphthene ring (Figure). The cyclopentane moiety (C1-C9-C8-C18-C11 ring) and the naphthalene moiety (C1-C10 ring) of the acenaphthene ring are almost coplanar (dihedral angle $\left.=2.06^{\circ} ; \mathrm{C} 1-\mathrm{C} 11-\mathrm{C} 18-\mathrm{C} 8=3.78(12)^{\circ}\right)$. The dihedral angles between fluorobenzene rings and the naphthalene ring are $55.62^{\circ}$ [C12-C17 ring; $\mathrm{C} 1-\mathrm{C} 11-\mathrm{C} 12-$ $\left.\mathrm{C} 13=-170.79(13)^{\circ}\right]$ and $86.70^{\circ}$ [C19-C24 ring; C8-C18-C19$\left.\mathrm{C} 20=124.74(13)^{\circ}\right]$, respectively. The dihedral angle between the best planes of the two fluorobenzene rings is $51.34(8)^{\circ}$. Three kinds of intramolecular non-covalent bonding interactions are observed: $\mathrm{O}-\mathrm{H} \cdots \mathrm{O}$ classical hydrogen bond

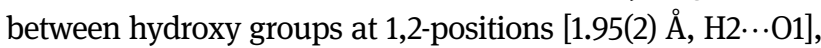
$\mathrm{C}-\mathrm{H}$... O non-classical hydrogen bond between $\mathrm{H} 2 \mathrm{O}$ atom of 2-fluorophenyl moiety and oxygen atom of hydroxy group ( $2.32 \AA, \mathrm{H} 2 \mathrm{O} \cdots 02$ ), and $\mathrm{C}-\mathrm{F} \cdots \pi$ interaction between F2 atom

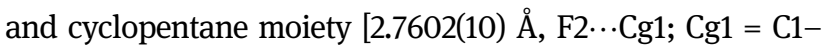
C9-C8-C18-C11 ring]. On the other 2-fluorophenyl group, the fluorine atom is disordered over two positions with site occupancies of 0.94 and 0.06 [F1A and F1B]. Therefore, the title compound is postulated as a mixture of two components, i.e., endo-exo (F2 and F1A/H17; major component) and endo-endo (F2 and F1B/H13; minor one) types. In the crystal packing, intermolecular classical hydrogen bond between hydroxy and methoxy groups links molecules into two-fold helical

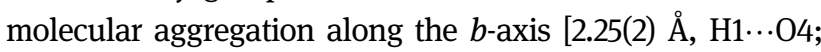
symmetry code: $3 / 2-x, 1 / 2+y, 1 / 2-z]$. The two-fold helical molecular aggregations are further gathered by two bidirectional forces: the $\mathrm{C}-\mathrm{H} \cdots \pi$ non-classical hydrogen bond between a methoxy group and the naphthalene ring along $c$-axis [2.66 $\AA$, H26B $\cdots . C$ Cg3; $\mathrm{Cg} 3=\mathrm{C} 5-\mathrm{C} 10$ ring; symmetry code: $2-\mathrm{x},-\mathrm{y},-\mathrm{z}]$ and a $\mathrm{C}-\mathrm{H} \cdots \mathrm{O}$ non-classical hydrogen bond between the $\mathrm{H} 22$ atom of the 2-fluorophenyl group and

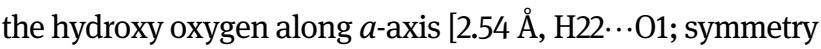
code: $-1 / 2+x, 1 / 2-y,-1 / 2+z]$. The hydroxy group $(02-\mathrm{H})$ that shares its hydrogen atom with the other hydroxy group (01-H) also makes a non-classical hydrogen bond with the hydrogen atom $(\mathrm{H}-\mathrm{C} 20)$ forming a warped sixfive-five fused tricyclic structure, at the end of which the fluorine atom (F2) occupying the opposite $o$-position intramolecularly interacts with the cyclopentane moiety. On the other phenyl group, the $o$-positioned hydrogen and fluorine atoms are free from effective interactions, which induces comparably stable two components as displayed in disorder of two atoms.

Acknowledgments: The authors express their gratitude to Mr Hirokazu Shiomichi and Mr Genta Takahara, Department of Organic and Polymer Materials Chemistry, Tokyo University of Agriculture and Technology, for their technical supports.

Author contributions: All the authors have accepted responsibility for the entire content of this submitted manuscript and approved submission.

Research funding: JSPS KAKENHI, grant number JP20K05473 and Tokyo Ohka Foundation for The Promotion of Science and Technology, grant number 216065.

Conflict of interest statement: The authors declare no conflicts of interest regarding this article.

\section{References}

1. Rigaku. PROCESS-AUTO. Rigaku Corporation, Tokyo, Japan (1998).

2. Rigaku. CRYSTALSTRUCTURE. Rigaku Corporation, Tokyo, Japan (2010).

3. Sheldrick G. M. A short history of SHELX. Acta Crystallogr. 2008, A64, 112-122.

4. Sheldrick G. M. Crystal structure refinement with SHELXL. Acto Crystallogr. 2015, C71, 3-8.

5. Burnett M. N., Johnson C. K. ORTEPIII Report ORNL-6895; Oak Ridgeational Laboratory: Tennessee, USA, 1996.

6. Mido T., litsuka H., Kobayashi M., Noguchi K., Yonezawa N., Okamoto A. Fluoro group pivoting dual hydrogen bonding intramolecular bridge for 1,2-bis(2-fluorophenyl) acenaphthenediol molecule in solution: NMR spectrometrical confirmation of simultaneous participation of $\mathrm{F}-\mathrm{C}\left(\mathrm{sp}^{2}\right)$ group to through-space-couplings with aromatic and hydroxy hydrogen atoms. Chem. Lett. 2020, 49, 295-298. 
7. Watanabe S., Nagasawa A., Okamoto A., Noguchi K., Yonezawa N. (2,7-Dimethoxynaphthalene-1,8-diyl)bis(4-fluorobenzoyl) dimethanone Acta Crystallogr 2008, E66, 0807.

8. Desiraju G. R. $\mathrm{C}-\mathrm{H} \cdots \mathrm{O}$ and other weak hydrogen bonds. from crystal engineering to virtual screening. Chem. Commun. 2005 , 24, 2995-3001.

9. Desiraju G. R. Hydrogen bridges in crystal engineering: interactions without borders. Acc. Chem. Res. 2002, 35, 565-573.

10. Desiraju G. R., Steiner T. The Weak Hydrogen Bond in Structural Chemistry and Biology; Oxford University Press: Oxford, 1999.

11. Taylor R., Kennard O. Crystallographic evidence for the existence of $\mathrm{C}-\mathrm{H} \cdots \mathrm{O}, \mathrm{C}-\mathrm{H} \cdots \mathrm{N}$, and $\mathrm{C}-\mathrm{H} \cdots \mathrm{Cl}$ hydrogen bonds. J. Am. Chem. Soc. 1982, 104, 5063-5070.

12. Okamoto A., Yonezawa N. Unique and specific reaction behavior and characteristic spatial organization of non-coplanarly aromatic-ring- accumulated molecular compounds. J. Synth. Org. Chem. 2015, 73, 339-360.

13. Muto T., lida K., Noguchi K., Yonezawa N., Okamoto A. Crystal structure and Hirshfeld surface analysis of 2-hydroxy-7-methoxy1,8-bis(2,4,6-trichlorobenzoyl)naphthalene. Acta Crystallogr. 2019, E75, 1418-1422.
14. Kobayashi M., lida K., litsuka H., Li K., Yonezawa N., Okamoto A. The crystal structure of 1,8-bis(4-methoxybenzoyl)naphthalene-2,7-diyl dibenzoate $\mathrm{C}_{40} \mathrm{H}_{28} \mathrm{O}_{8}$. Z. Krystallogr. N. Cryst. Struct. 2021, 236, 945-947.

15. Iida K., Sakamoto R., Li K., Kobayashi M., litsuka H., Yonezawa N., Okamoto A. Crystal structure of bis(1,8-dibenzoyl-7-

methoxynaphthalen-2-yl)terephthalate: terephthalate phenylene moiety acts as biacceptor of bidirectional $\mathrm{C}-\mathrm{H} \cdots \pi$ non-classical hydrogen bonds. Eur. J. Chem 2021, 12, 147-153.

16. litsuka H., Li K., Kobayashi M., lida K., Yonezawa N., Okamoto A. Crystal structure of 1,2-bis(4-fluorophenyl)-1-hydroxy-2,3,8trimethoxyacenaphthene: formation of a five-membered intramolecular $\mathrm{O}-\mathrm{H} \cdots \mathrm{O}$ hydrogen-bonded ring. Acta Crystallogr. 2021, E77, 175-179.

17. Mido T., litsuka H., Yokoyama T., Takahara G., Ogata K., Yonezawa N., Okamoto A. Crystal Structure of (1R,2S)-1,2bis(4-chlorophenyl)-3,8-dimethoxyacenaphthene-1,2-diol: tetrameric string of four conformers connected by classical hydrogen bonds and molecular accumulation alignment by linking of the tetramers with the aid of non-classical hydrogen bonds. Eur. Chem. Bull. 2017, 6, 273-280. 